\section{Ueber den Einfluss der salinischen Laxantia auf den Stoffwechsel.}

Vortray in der balneologrischen Section der Gesellschaft für Heilkunde aul 25. Jaunar 1879 .

rou

\section{W. Znelzer.}

M. H.! Lch erlaube mir Ihre Anfuerksamkeit anf die Wirknngen zu richten, weiche die salinischen Laxantia auf die Vorgänge des Stoffwechsels ansüben. Es giebt kanm ein Medicament, welches in der täglichen Praxis wie in der Balneologie so lıänfig angewandt wird, keines, desseu Auweudung so altbekannt und dessen wohlthätiger Einflnss in maunigfachen lirankheitsformen so unbezweifelt ist, wie die Mittolsalze. Denuoch ist nusere Kenutuiss vou den physiologischen Forgängen, die hierbei mitwirken, so überaus geriug, dass wir wahrlich hierans allein hiureichenden Grund zu der Frage liaben, ob die bisher übliche Art der Untersuchung dieser Verhältuisse zwechmässic ist o(ter ob nicht schon in der Art nud Weise der Fragestellıng die Selwwierirbeit fïr eine rationelle Lösnıg liegt.

Die Frage zerfällt nugerwnugen in zwei Thenle: Anf welche Welse komıt die Abfulırwirhung zn Stande? Welchen Einfluss hat das Laxireu auf deu Stoffwechsel?

So lauge es als eine Art von Dogma galt, dass die Laxautien und gan besonders die salinischen eiue profuse Trinssudation in das Lumen des Darus veraulassen, hielt man sich von der genaneren Lintersuchungr der Abfïlıwirhung jener Mittel fern, weil durch diese Aunahne anscheineud alles erklärt war. Liebigr, dessen grosse Bedentnng wie in vieltun anderen Geljeten so anch in der Medicin einen ïberwältigenden Einfhıs ausible, trus wesentlich dazn bei, der Abfiihrwirknne einen rein plysikalisch erklärharen Charakter anfzudrücken. Lr wies bei Gelegenhe!l der Lntersuchun ren über die Mineralquellen zn Soden daranf hin, dass manche Stoffe die Eirceuschaft haben deu Körpergeweben Wasser zn entziehen nud bezeichuete dies als die Lrsache der laxirenden Wirkung einiger Salze. Den Grund suchte er darin, dass die Flïssigkeiten, welche einen grösseren Salzgehalt besitzen als das Blut, dieseu Wasser entziehen, das nuit den Fäcalmassen veruischt durch den Darm ansgesthieden wird. Je concentrirter also eine Salzlösung ist, ıuı so kräliticrer abfïlıreul mïsse sie deshalb wirkeu.

So logisch eine solche Schlıssfolgeruıg erscheinen muss, wenn man es unr mit todten Meinbranen zu thun hat. wie sie dem Cliemiker zu Gebote stehen, so ergaly sich sofort ihre Culıaltbarkeit, als sich ein Physiologe mit der Frage beschäfigte. Aubert fand, im Gegensatz z.n jeuer apriorisuschen Anuahne, dass die Concentration der Salzlösuner keinen Einfluss ant ihre abführende Wirkung hat, und dass sogar ausserordentlich diluirte Lösungen von Glanbersalz nnd Bittersalz die gleiche Wirlinng erzielen wie concentrirte. Die Abfïhrwirknug ist deshalb unr als Folge der Steigerung der Peristaltik zn betrachten, welche durch Reizung der Nerveu liervorgerufen wird.

Diese Ausiclıt erst wurde zum Ansqangspunkt der weiteren Untersuchungeu. Weun man eine Daruschlinge ans ihrem Znsammenhange init dem ülırigen Darm löst, die dazu gehörigen Nerven nud Gefässe aber intact lässt, so rufeu, wie Thiry, Schiff n. a. zeigten, weder Drastika in kleineren Dosen noch Mittelsalze in verdünnten Lösıngen in dieselben eingespritzt darin eine Traussudation von Blutfiissigkeit oder verstärhte Secretion der Darmsäfte hervor. Dagegen tritt ein bedentender Erguss von Flüssigkeit ein, sobald der Einfluss der Nerven - durch Durchschneidung - anfgehoben wird (M oreau).

Die stärksten Abfülırmittel, z. B. Crotouöl, bleiben bei innerlicher Darreichung ohue Wirkung uach Durchsehneidung der beiden Halsvagi. Feruer ruft die Injection auch rou bedeutenden Mengen Glanbersala
(50 Gruı.) in die Jugularvene vou Hunden nach Buchheım keine Diarrhoe hervor. -

Dagerren sehen wir bei innerlicher Auweudung von Crotonöl Diarrloe schon zu einer Zelt auftreteu, wo das Mittel noch im Magen liegt (Radzicjewsky) und inı Gegeusatz. dazı die abfiihrende Wirknng der Mittelsalze nuehr oder weniger anfgehoben, sobald, wie Buch heim zeırte, ihuen Opiate oder Gerbsäure beigefügt werdeu.

Wil nüssen deshalb aunehmen und zahlreiche directe Versuche sprechen ebenfalls dafür, dass die uächste Wirhung dor laxantia anl einer localisirten Reiznng der Magennerven berulıt, wodurch ene reflec-

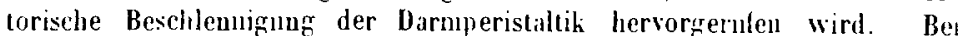
Anwendmig solcler Mittel (Gnmmi gutti, Jalapa, Scammonum, Aloë etc.), welche zn ilırer Lösıme der alkalinıschen säfte (Galle unt andere Darmsäfte) ledürfen, wird der gleiche Effect erst dann bewirkl, weun sic mit diesen Säften in Berührnug gehomuen sind, durch directe Reizungr der Darnwand und ihrer Ganglien.

Wir kömen uns also vorstellen, dass die evalinireude barrlooenacheude Wirkung der Laxantia wesentlich dadurch hervorarerufen wird, dass die Darmsäfte, welche in den oberen Theil des lyarmirohrs alıs den Paukreas und delı Darmdrüsen ergossen werden, nocht wie im intacten Zustande nuehr oder wenicrer vollständig resorbirt, souderu schuell durch den Darm hindurchgetrieljen nud ansgesclieden werden ${ }^{3}$ ).

Wenn wir in diesen Resultaten das maassgelende Monent suchen mïssen, so haben wir darin einen directen Anscrangspunkt für die Untersnchmngr über die Veräuderungen, welche die Laxantien im Stuffwechsel bewirken. lst die Theorie richtig, so müssen die Verhältuisse des Stoffwechsels ilır entsprechen. Wäre dies nicht der Fall. lıesson die Verhältuisse des Stoffwecbsels lieine Lebereinstimunnng damut erkeunen, so wäre ladurch zahleumässig bewiesen, dass nusere theoretische Ausicht falsch oder mudesteus uur enuscitiog richltg ist.

Was wissen wir iiber die Verändernngren, welche del Stoffwechsel durch die laxirende Wirkıngr der Mıttelsalze eriährt?

Sic werden unir zugebeu, dass unsere kemntuiss hierïher nngemein bescheiden ist, wenn ich Ihnen sage, dass sie im Wesenthelien nur durch zwei Arbetten begrïndel ist, von denen noch dazn de zwelte die Resultate der vorherchehenden in Fracre stellt.

Die beiden Arbeiten sind von Seegen mnd Voit. Der erstere ıneiut, dass der Gennss von Glaubersalz den Unsat\% der Eiweisshörper in thierischen Organismus lierabsetzt; Voit Jestreitet dies nnd findet deu Eiweissmmsatz durch de Einführung der Mittelsalze nicht verändert. Erst bei längerem Fortgebranch tritt ein dem Hungern ähulicher Zustand ein (Aluahne des Fettoolsters etc.), weil weniger Nahrullg zur Anfuahme gelangt. -

Wie Sie selucn, bentheilen beide Arbeiten den Eiweissmmat\% des Urganismus. Es wäre grwiss ein wichtiges Resultat, weun wenigstens due Grösse des Lmsatzes eines so wichtigen hörperbestandtheils auch unr amähernd ıud im Bausch nud Bagen ermittelt werden hö̈nte.

lch muss indessen bestreiten, dass dies anf dem lusher enneschlagenen Wege ïberhiupt möglich ist. Ausser den ill meiner Mhamblhug: „Zur Statik des Stoflweclusels" ${ }^{2}$ ) angefiihrten scheinen nir hiertiil liesonders folgende Ciriinde dafür maassarebend. Jenes Ĺrtheil stützt sich anf de Beobachung des liörpergewchts nud des in den Exkreten erscheinenden Stickstoffs mid den Vercrleich desselben nit den in der Nahrung enureführten Stichstoffmengeu. - Die Bedentung des Körpergewichts für solche Znecke hat loit später selbst melırfach abfällig bemtheilt, so dass es lierbei eine besondere Wichtigkeit nicht haben kann.

Es bleiben also unr die Stickstoflmengen, $11 n$ damit deu limsit: der Eiweisskörper festzustelleu.

Wie wenig indess time solche Schlussfolgerung berechıgt ist. mören folgende Betrachtungen zeigen.

Wenn die Weichtheile des Thierköruers, anf die es be den lintersuchungeu über den Stoflwechsel wesentlich autioumu, gleichmässig ans Eiweissstoffen beständen, so köunte mau wobl annelmnen, dass aller Stichstoff, der in den Lxkreten gefunden wird, aus der Zersetzung dieser Stoffe hervorgegangen sei. Sie enthalten in ihrer mittleren Zusammen-

1) In einem gewissen Gegeusatz zu dieser Amahme hat zwar Brieger, dessen Resultate sonst mit den oben citirten ibereinstimmen. in einem seiner Versuche gefunden, dass eine concentrirte 20 procentige) Lösung ron Bittersalz. abweichend ron anderu Drasticis in eiuem abeschlos senen Darnstück auch eine vermehrte Secretion hervorruft Indessen ist dieser Versuch unter eiuer starken Morphiumwirkung vorgenunmen, wodurch unzweifelhaft die Darmnerven erheblich beeinflusst werden. Wahrscheinlich hat anch die concentrirte Salzlosing an und für sich eine lähmende Wirkung auf die Nerven der Dalmwand. Für die dllgemeine Theorie der Abführwirkung ist jedenfalls dieser Versuch deshalb nicht maassgebend, weil wir in praxi nicht mit derartig concen. trirten Lösungen arbeiten. Die Mineralwässer von Carlsbad und Marien. bad z. B. enthalten kaum 2 Proc. Glaubersalz und im Ganzen nur etwa 4 Proc. fester Bestandtheile.

2) Beitrăge zur Medicinal-Statistik, von Schweig, Schwartz und Zuelzer, Heft 3, pag. 101 
setzung etwa 16 Proc. Stickstoff; es wäre also die Annahme plausibel, sobald man $16 \mathrm{Grm}$. Stickstoff $(=34,2 \mathrm{Grm}$. Harustoff $)$ unter den Ausscheidungsproducten fãnde, dass $100 \mathrm{Grm}$. Eiweissstoffe von den Körperhestandtheilen zerfallen seien.

Freilich würde ein Chemiker es sehr bedenklich finden, wenn man ihm sagen wollte, Körper, die so complicirt sind, wie die Eiweissstoffe, seien durch einen einzigen Bestandtheil charakterisirt; selbst in Fällen, wo ein Zweifel nicht gut möglich wäre, würde ein solches Verfahren den wissenschaftlichen Anforderungen nicht genügen.

Nun haben wir es aber gar nicht ausschliesslich mit Albuminaten 7.u thun. Der Thierkörper besteht zwar zum grössten Theil daraus (namentlich die Muskeln); ausserdem aber giebt es sehr wichtige Organgruppen, besonders das ganze Nervensystem, in denen die Eiweisskörper ganz wesentlich zurücktreten, deren Hauptbestandtheil vielmehr dass ebenfalls Stickstoff-haltige Lecithin bildet. Wie der Stoffumsatz in diesen Organen vor sich gelit, ist nicht bekannt; dass er conform den Veränderungen eintritt, welchen die Muskeln unterliegen, darf schon deshalb nicht angenommen werden, weil alle physiologischen und chemischen Erfahrungen dafür sprechen, dass die Alterationen im Stoffwechsel der Muskeln abhängig sind von nervösen Einflüssen.

Wird also, wie es von $V_{0}$ it und seinen Schülern geschieht, pars pro toto gesetz.t, indem der gesammte ausgeschiedene Stickstoff als von Eiweisskörpern herrührend angesehen wird, so wird ein Fehler in die Rechnung eingeführt, der schon deshalb unmessbar wird, weil wir gar nicht wissen, ob nicht der Stoffumsatz in den Lecithin-reichen Geweben viel lebendiger ist, als in den Muskeln?

(Schluss folgt.) 